\title{
Stabilities for Nonisentropic Euler-Poisson Equations
}

\author{
Ka Luen Cheung and Sen Wong \\ Department of Mathematics and Information Technology, The Hong Kong Institute of Education, 10 Lo Ping Road, \\ Tai Po, New Territories, Hong Kong \\ Correspondence should be addressed to Sen Wong; senwongsenwong@yahoo.com.hk
}

Received 6 December 2014; Revised 8 March 2015; Accepted 9 March 2015

Academic Editor: Carlo Bianca

Copyright ( $\odot 2015$ K. L. Cheung and S. Wong. This is an open access article distributed under the Creative Commons Attribution License, which permits unrestricted use, distribution, and reproduction in any medium, provided the original work is properly cited.

We establish the stabilities and blowup results for the nonisentropic Euler-Poisson equations by the energy method. By analysing the second inertia, we show that the classical solutions of the system with attractive forces blow up in finite time in some special dimensions when the energy is negative. Moreover, we obtain the stabilities results for the system in the cases of attractive and repulsive forces.

\section{Introduction}

The compressible nonisentropic Euler $(\delta=0)$ or EulerPoisson $(\delta= \pm 1)$ system for fluids can be written as

$$
\begin{gathered}
\rho_{t}+\nabla \cdot(\rho u)=0, \\
(\rho u)_{t}+\nabla \cdot(\rho u \otimes u)+\nabla P+\beta \rho u=-\delta \rho \nabla \Phi, \\
S_{t}+u \cdot \nabla S=0, \\
\Delta \Phi(t, x)=\alpha(N) \rho, \\
P=K \rho^{\gamma} e^{S},
\end{gathered}
$$

where $\beta \geq 0$ is the frictional damping constant and $\alpha(N)$ is a constant related to the unit ball in $\mathbb{R}^{N} \cdot \alpha(1)=2, \alpha(2)=2 \pi$ and

$$
\alpha(N)=N(N-2) V(N),
$$

where $V(N)$ is the volume of the unit ball in $\mathbb{R}^{N}$. As usual, $\rho=\rho(t, x) \geq 0, u=u(t, x) \in \mathbb{R}^{N}$, and $S(t, x)$ are the density, the velocity, and the entropy, respectively. $P$ is the pressure function, for which the constants $K \geq 0$ and $\gamma \geq 1$.

When $\delta=1$, the system is self-attractive. The system (1) is the Newtonian description of gaseous stars [1]. When $\delta=-1$, the system comprises the Euler-Poisson equations with repulsive forces and can be used as a semiconductor model $[2,3]$.
When $\delta=0$, the system comprises the compressible Euler equations and can be applied as a classical model in fluid mechanics [3]. For more classical and recent results in these systems, readers can refer to [1,4-10].

It is well known that the solution for the Poisson equation $(1)_{4}$ can be written as

$$
\Phi(t, x)=\alpha(N) \int_{\mathbb{R}^{N}} G(x-y) \rho(t, y) d y,
$$

where $G$ is the Green's function for the Poisson equation in the $N$-dimensional spaces defined by

$$
G(x):= \begin{cases}|x|, & N=1 ; \\ \log |x|, & N=2 ; \\ \frac{-1}{|x|^{N-2}}, & N \geq 3 .\end{cases}
$$

Notation. In the following discussion, classical solutions $(\rho, u, S)$ are $C^{1}$ solutions with compact support $\Omega=\Omega(t)$ for each fixed time $t$. We also denote the total mass by $M$, where

$$
M=\int_{\Omega} \rho d x=\int_{\Omega(0)} \rho_{0} d x,
$$

where $\rho_{0}=\rho_{0}(x):=\rho(0, x)$.

Lastly, we will denote

$$
R(t)=\text { the diameter of } \Omega(t) .
$$




\section{Lemmas}

In this section, we establish some lemmas for the proof of the main results. The following lemma will be used to derive the energy functional for $\gamma>1$; namely,

$$
E(t)=\int_{\Omega}\left(\frac{1}{2} \rho|u|^{2}+\frac{1}{\gamma-1} P\right) d x+\frac{\delta}{2} \int_{\Omega} \rho \Phi d x
$$

is conserved in time if the system (1) is not damped.

Lemma 1. For the classical solution $(\rho, u, S)$ of system (1), we have

$$
\int_{\Omega} P_{t} d x=(\gamma-1) \int_{\Omega} u \cdot \nabla P d x
$$

where $P$ is defined by $(1)_{5}$.

Proof. We have

$$
\begin{aligned}
P_{t} & =K\left(\rho^{\gamma} e^{S} S_{t}+e^{S} \gamma \rho^{\gamma-1} \rho_{t}\right) \quad \text { by }(1)_{5} \\
& =P S_{t}+K e^{S} \gamma \rho^{\gamma-1}[-\nabla \cdot(\rho u)] \quad \text { by }(1)_{5} \text { and }(1)_{1} \\
& =P(-u \cdot \nabla S)+K e^{S} \gamma \rho^{\gamma-1}[-\nabla \cdot(\rho u)] \quad \text { by }(1)_{3} \\
& =-P\left(\sum_{i=1}^{N} u_{i} \partial_{i} S\right)-K \gamma e^{S} \rho^{\gamma-1}\left[\sum_{i=1}^{N}\left(\rho \partial_{i} u_{i}+u_{i} \partial_{i} \rho\right)\right] \\
& =-\gamma P \sum_{i=1}^{N} \partial_{i} u_{i}-\sum_{i=1}^{N}\left[P \partial_{i} S+K \gamma e^{S} \rho^{\gamma-1} \partial_{i} \rho\right] u_{i} \quad \text { by }(1)_{5} \\
& =-\gamma P \sum_{i=1}^{N} \partial_{i} u_{i}-\sum_{i=1}^{N}\left(\partial_{i} P\right) u_{i} \\
& =-\gamma P \nabla \cdot u-u \cdot \nabla P .
\end{aligned}
$$

Note that, by Divergence Theorem,

$$
-\int_{\Omega} P \nabla \cdot u d x=\int_{\Omega} u \cdot \nabla P d x .
$$

Thus,

$$
\int_{\Omega} P_{t} d x=(\gamma-1) \int_{\Omega} u \cdot \nabla P d x
$$

Next, the results of the following two lemmas will be used in the derivations of the energy functionals for both $\gamma>1$ and $\gamma=1$. It will be shown that in Section 3 the energy functional for $\gamma=1$ is

$$
E(t)=\int_{\Omega}\left(\frac{1}{2} \rho|u|^{2}+K \rho e^{S}(\ln \rho-1)\right) d x+\frac{\delta}{2} \int_{\Omega} \rho \Phi d x
$$

Lemma 2. For the classical solution $(\rho, u, S)$ of system (1), we have

$$
\begin{aligned}
\int_{\Omega}\left(\frac{1}{2} \rho|u|^{2}\right)_{t} d x= & -\int_{\Omega} u \cdot \nabla P-\delta \int_{\Omega}(\nabla \Phi) \cdot(\rho u) d x \\
& -\beta \int_{\Omega} \rho|u|^{2} d x
\end{aligned}
$$

where $P$ is defined by $(1)_{5}$ and $\Phi$ is the solution of $(1)_{4}$.

Proof. We have

$$
\begin{aligned}
\left(\frac{1}{2} \rho|u|^{2}\right)_{t} & \\
= & (\rho u)_{t} \cdot u-\frac{1}{2} \rho_{t}|u|^{2} \quad \text { (by product rule) } \\
= & -(\delta \rho \nabla \Phi+\nabla \cdot(\rho u \otimes u)+\nabla P+\beta \rho u) \cdot u \\
& +\frac{1}{2}(\nabla \cdot(\rho u))|u|^{2} \quad \text { by }(1)_{1} \text { and }(1)_{2} .
\end{aligned}
$$

One can check a detail proof of the following equality in the Appendix:

$$
\int_{\Omega}\left(\frac{1}{2}|u|^{2} \nabla \cdot(\rho u)-u \cdot[\nabla \cdot(\rho u \otimes u)]\right) d x=0 .
$$

Thus,

$$
\int_{\Omega}\left(\frac{1}{2} \rho|u|^{2}\right)_{t} d x=-\int_{\Omega}(\delta \rho \nabla \Phi+\nabla P+\beta \rho u) \cdot u d x
$$

by (16) and (17).

Thus, the proof is complete.

Lemma 3. For the classical solution $(\rho, u, S)$ of system (1), we have

$$
\int_{\Omega}(\nabla \Phi) \cdot(\rho u) d x=\int_{\Omega} \Phi \rho_{t} d x=\frac{1}{2} \int_{\Omega}[\rho \Phi]_{t} d x,
$$

where $\Phi$ is the solution of $(1)_{4}$.

Proof. We have

$$
\begin{aligned}
\int_{\Omega}(\nabla \Phi) \cdot(\rho u) d x \\
\quad=-\int_{\Omega} \Phi \nabla \cdot(\rho u) d x \quad \text { by Divergence Theorem } \\
\quad=\int_{\Omega} \Phi \rho_{t} d x \text { by }(1)_{1} .
\end{aligned}
$$

Thus, the first equality in (19) holds.

Next,

$$
\begin{aligned}
\int_{\Omega} \Phi \rho_{t} d x & =\frac{1}{\alpha(N)} \int_{\Omega} \Phi \Delta \Phi_{t} d x \quad \text { by }(1)_{4} \\
& =\frac{1}{\alpha(N)} \int_{\Omega} \Delta \Phi \Phi_{t} d x \quad \text { by Green's Formula } \\
& =\int_{\Omega} \rho \Phi_{t} d x \quad \text { by }(1)_{4} .
\end{aligned}
$$

Thus, the second equality in (19) holds. 
The lemma below is crucial to obtaining the energy functional for $\gamma=1$. Comparing the left hand sides of (8) and (22), we note that the left hand side of (22) (given in the next lemma), which contains the term $\ln \rho-1$, is nontrivial to be found.

Lemma 4. For the classical solution $(\rho, u, S)$ of system (1) with $\gamma=1$, we have

$$
\frac{\mathrm{d}}{\mathrm{d} t} \int_{\Omega} K \rho e^{S}(\ln \rho-1) d x=\int_{\Omega} u \cdot \nabla P d x,
$$

where $P$ is defined by $(1)_{5}$.

Proof. Note that

$$
\begin{aligned}
& {\left[\rho e^{S}(\ln \rho-1)\right]_{t}} \\
& \quad=e^{S}[\rho(\ln \rho-1)]_{t}+[\rho(\ln \rho-1)] e^{S} S_{t} \\
& \quad=e^{S}\left[\rho_{t}+(\ln \rho-1) \rho_{t}\right]+e^{S} \rho(\ln \rho-1) S_{t} \\
& =e^{S}(\ln \rho) \rho_{t}+e^{S} \rho(\ln \rho-1) S_{t} \\
& =-e^{S}(\ln \rho)(\nabla \cdot(\rho u))-e^{S} \rho(\ln \rho-1)(u \cdot \nabla S) \\
& \text { by }(1)_{1} \text { and }(1)_{3} .
\end{aligned}
$$

Thus,

$$
\begin{aligned}
\frac{\mathrm{d}}{\mathrm{d} t} \int_{\Omega} & K \rho e^{S}(\ln \rho-1) d x \\
= & -\int_{\Omega} K e^{S}(\ln \rho)[\nabla \cdot(\rho u)] d x \\
& -\int_{\Omega} K e^{S} \rho(\ln \rho-1)(u \cdot \nabla S) d x \\
= & \int_{\Omega}(\rho u) \cdot \nabla\left[K e^{S}(\ln \rho)\right] d x \\
& -\int_{\Omega} K e^{S} \rho(\ln \rho-1)(u \cdot \nabla S) d x \\
= & \int_{\Omega} u \cdot\left[K \rho \nabla\left(e^{S} \ln \rho\right)-K e^{S} \rho(\ln \rho-1) \nabla S\right] d x \\
= & \int_{\Omega} u \cdot\left[K e^{S} \nabla \rho+K e^{S} \rho \nabla S\right] d x \\
= & \int_{\Omega} u \cdot \nabla P d x \quad \text { by }(1)_{5} .
\end{aligned}
$$

The proof is complete.

\section{Main Results}

In this section, we find out the energy functionals for the system (1) in the case of $\gamma>1$ (Proposition 5) and $\gamma=1$ (Proposition 6). Moreover, we establish the stabilities results (Proposition 8) and a blowup result (Proposition 9) for system (1).
Proposition 5. For the classical solution $(\rho, u, S)$ of system (1) with $\gamma>1$, let

$$
E(t)=\int_{\Omega}\left(\frac{1}{2} \rho|u|^{2}+\frac{1}{\gamma-1} P\right) d x+\frac{\delta}{2} \int_{\Omega} \rho \Phi d x .
$$

Then,

$$
\dot{E}(t)=-\beta \int_{\Omega} \rho|u|^{2} d x,
$$

where $\dot{E}(t)$ is the devertive of $E(t)$ with respect to $t$.

Thus, $E(t)$ is a decreasing function and is conserved if the system is not damped.

Proof. By Lemma 1,

$$
\frac{1}{\gamma-1} \int_{\Omega} P_{t} d x=\int_{\Omega} u \cdot \nabla P d x
$$

By Lemma 2,

$$
\begin{aligned}
\int_{\Omega}\left(\frac{1}{2} \rho|u|^{2}\right)_{t} d x= & -\int_{\Omega} u \cdot \nabla P-\delta \int_{\Omega}(\nabla \Phi) \cdot(\rho u) d x \\
& -\beta \int_{\Omega} \rho|u|^{2} d x .
\end{aligned}
$$

By Lemma 3,

$$
\int_{\Omega}(\nabla \Phi) \cdot(\rho u) d x=\frac{1}{2} \int_{\Omega}[\rho \Phi]_{t} d x .
$$

Thus, the proof is complete.

Proposition 6. For the classical solution $(\rho, u, S)$ of system (1) with $\gamma=1$, let

$$
E(t)=\int_{\Omega}\left(\frac{1}{2} \rho|u|^{2}+K \rho e^{S}(\ln \rho-1)\right) d x+\frac{\delta}{2} \int_{\Omega} \rho \Phi d x .
$$

Then,

$$
\dot{E}(t)=-\beta \int_{\Omega} \rho|u|^{2} d x,
$$

where $\dot{E}(t)$ is the derivative of $E(t)$ with respect to $t$.

Thus, $E(t)$ is a decreasing function and is conserved if the system is not damped.

Proof. By Lemma 2,

$$
\begin{aligned}
\int_{\Omega}\left(\frac{1}{2} \rho|u|^{2}\right)_{t} d x= & -\int_{\Omega} u \cdot \nabla P-\delta \int_{\Omega}(\nabla \Phi) \cdot(\rho u) d x \\
& -\beta \int_{\Omega} \rho|u|^{2} d x .
\end{aligned}
$$

By Lemma 3,

$$
\int_{\Omega}(\nabla \Phi) \cdot(\rho u) d x=\frac{1}{2} \int_{\Omega}[\rho \Phi]_{t} d x .
$$

By Lemma 4,

$$
\frac{\mathrm{d}}{\mathrm{d} t} \int_{\Omega} K \rho e^{S}(\ln \rho-1) d x=\int_{\Omega} u \cdot \nabla P d x .
$$

Thus, the proof is complete. 
Proposition 7. Let

$$
H(t)=\int_{\Omega} \rho|x|^{2} d x
$$

We have

$$
\begin{gathered}
\ddot{H}(t)=-\beta \dot{H}(t)+2 \int_{\Omega}\left(\rho|u|^{2}+2 P\right) d x-2 \pi \delta M^{2}, \\
\qquad \text { for } N=2, \\
\ddot{H}(t)=-\beta \dot{H}(t)+2 \int_{\Omega}\left(\rho|u|^{2}+N P\right) d x \\
+(N-2) \delta \int_{\Omega} \rho \Phi d x, \quad \text { for } N \geq 3,
\end{gathered}
$$

where $\dot{H}(t)=(\mathrm{d} / \mathrm{d} t) H(t), \ddot{H}(t)=(\mathrm{d} / \mathrm{d} t) \dot{H}(t),(\rho, u, S)$ is a classical solution of system (1), and $M$ is defined by (5).

Proof.

$$
\begin{aligned}
\dot{H}(t) & =-\int_{\Omega} \nabla \cdot(\rho u)|x|^{2} d x \quad \text { by }(1)_{1} \\
& =\int_{\Omega} \nabla|x|^{2} \cdot(\rho u) d x \quad \text { by Divergence Theorem } \\
& =\int_{\Omega} 2 x \cdot(\rho u) d x, \\
\ddot{H}(t) & =2 \int_{\Omega} x \cdot(-\nabla \cdot(\rho u \otimes u)-\nabla P-\delta \rho \nabla \Phi-\beta \rho u) d x \\
& =-\beta \dot{H}(t)+2 \int_{\Omega} x \cdot(-\nabla \cdot(\rho u \otimes u)-\nabla P-\delta \rho \nabla \Phi) d x .
\end{aligned}
$$

We split the last term of the above equality into three parts.

Firstly,

$$
\begin{aligned}
-\int_{\Omega} & x \cdot[\nabla \cdot(\rho u \otimes u)] d x \\
& =-\sum_{j=1}^{N} \int_{\Omega} x_{j} \sum_{i=1}^{N} \partial_{i}\left(\rho u_{i} u_{j}\right) d x \quad \text { by definitions } \\
& =\int_{\Omega} \rho|u|^{2} d x \quad \text { by integration by parts. }
\end{aligned}
$$

Secondly,

$$
\begin{aligned}
-\int_{\Omega} x \cdot \nabla P d x & =\int_{\Omega} P \nabla \cdot x d x \quad \text { by Divergence Theorem } \\
& =\int_{\Omega} N P d x
\end{aligned}
$$

Thirdly,

$$
\begin{aligned}
& -\delta \int_{\Omega} \rho x \cdot \nabla \Phi d x \\
& \quad=-\delta \alpha(N) \int_{\Omega} \int_{\Omega} \rho(x) \rho(y)\left[\nabla_{x} G(x-y) \cdot x\right] d y d x \\
& \quad=:-\delta \alpha(N) I,
\end{aligned}
$$

where $\nabla_{x}$ is the gradient operator with respect to the spatial variable $x$.

Note that

$$
\begin{aligned}
I= & \int_{\Omega} \int_{\Omega} \rho(x) \rho(y)\left[\nabla_{x} G(x-y) \cdot x\right] d y d x \\
= & \int_{\Omega} \int_{\Omega} \rho(x) \rho(y)\left[\nabla_{x} G(x-y) \cdot(x-y)\right] d y d x \\
& +\int_{\Omega} \int_{\Omega} \rho(x) \rho(y)\left[\nabla_{x} G(x-y) \cdot y\right] d y d x .
\end{aligned}
$$

For $N=2$,

$$
\nabla_{x} G(x)=\nabla_{x} \log |x|=\frac{1}{|x|^{2}} x
$$

Thus,

$$
\begin{aligned}
& I=\int_{\Omega} \int_{\Omega} \rho(x) \rho(y) d y d x-I, \\
& I=\frac{1}{2} \int_{\Omega} \int_{\Omega} \rho(x) \rho(y) d y d x=\frac{M^{2}}{2} .
\end{aligned}
$$

The result for $N=2$ is established.

For $N \geq 3$,

$$
\nabla_{x} G(x)=-\nabla_{x} \frac{1}{|x|^{N-2}}=(N-2) \frac{1}{|x|^{N}} x .
$$

Thus,

$$
\begin{gathered}
I=\int_{\Omega} \int_{\Omega} \rho(x) \rho(y)\left[\nabla_{x} G(x-y) \cdot(x-y)\right] d y d x \\
+\int_{\Omega} \int_{\Omega} \rho(x) \rho(y)\left[\nabla_{x} G(x-y) \cdot y\right] d y d x \\
=-\frac{(N-2)}{\alpha(N)} \int_{\Omega} \rho \Phi d x-I, \\
I=-\frac{N-2}{2 \alpha(N)} \int_{\Omega} \rho \Phi d x .
\end{gathered}
$$

The results for $N \geq 3$ are also established.

Now, we are ready to present the stability results.

Proposition 8. Considering the classical solutions of system (1), we have the following. 
Case 1. For $\delta=1, \beta=0, N=3$ or $4, \gamma \geq 2(N-1) / N$, and $E(0) \geq 0$, we have

$$
\lim _{t \rightarrow \infty} \inf \frac{R(t)}{t} \geq \sqrt{\frac{(N-2) E(0)}{M}} .
$$

Case 2. For $\delta=-1, \beta=0, N \geq 4$, and $\gamma \geq(N+2) / N$, we have

$$
\lim _{n \rightarrow \infty} \inf \frac{R(t)}{t} \geq \sqrt{\frac{2 E(0)}{M}} .
$$

Case 3. For $\delta=-1, \beta=0, N=2$, and $\gamma>1$, we have

$$
\lim _{n \rightarrow \infty} \inf \frac{R(t)}{t} \geq \sqrt{\pi M}
$$

Proof. First of all, by definitions (35), (6), and (5), we always have

$$
H(t)=\int_{\Omega(t)} \rho|x|^{2} d x \leq R^{2}(t) M
$$

Case 1. By Propositions 5 and 7, we have

$$
\begin{aligned}
\ddot{H}(t)= & 2 \int_{\Omega}\left(\rho|u|^{2}+N P\right) d x+(N-2) \int_{\Omega} \rho \Phi d x \\
= & 2 \int_{\Omega} \rho|u|^{2} d x+2 N \int_{\Omega} P d x \\
& +(N-2)\left[2 E(t)-\frac{2}{\gamma-1} \int_{\Omega} P d x-\int_{\Omega} \rho|u|^{2} d x\right] \\
= & {[2-(N-2)] \int_{\Omega} \rho|u|^{2} d x } \\
& +\left[2 N-\frac{2(N-2)}{\gamma-1}\right] \int_{\Omega} P d x+2(N-2) E(t) \\
\geq & 2(N-2) E(t) \\
= & 2(N-2) E(0) .
\end{aligned}
$$

Thus,

$$
H(t) \geq(N-2) E(0) t^{2}+\dot{H}(0) t+H(0) .
$$

In view of inequality (49), we have

$$
\frac{R(t)}{t} \geq \sqrt{\frac{(N-2) E(0)}{M}+\frac{\dot{H}(0)}{M t}+\frac{H(0)}{M t^{2}}} .
$$

Thus,

$$
\lim _{t \rightarrow \infty} \inf \frac{R(t)}{t} \geq \sqrt{\frac{(N-2) E(0)}{M}} .
$$

Case 2. By Propositions 5 and 7, we have

$$
\begin{aligned}
\ddot{H}(t)= & 2 \int_{\Omega}\left(\rho|u|^{2}+N P\right) d x+(N-2) \int_{\Omega} \rho(-\Phi) d x \\
= & 2\left[2 E(t)-\frac{2}{\gamma-1} \int_{\Omega} P d x-\int_{\Omega} \rho(-\Phi) d x\right] \\
& +2 N \int_{\Omega} P d x+(N-2) \int_{\Omega} \rho(-\Phi) d x \\
\geq & 4 E(t) \\
= & 4 E(0) .
\end{aligned}
$$

Note that $-\Phi$ is a positive function for $N \geq 3$ by (3) and (4). Thus,

$$
H(t) \geq 2 E(0) t^{2}+\dot{H}(0) t+H(0) .
$$

It follows that

$$
\lim _{n \rightarrow \infty} \inf \frac{R(t)}{t} \geq \sqrt{\frac{2 E(0)}{M}}
$$

Case 3. By Proposition 7, we have

$$
\begin{aligned}
\ddot{H}(t) & =2 \int_{\Omega}\left(\rho|u|^{2}+2 P\right) d x+2 \pi M^{2} \\
& \geq 2 \pi M^{2}
\end{aligned}
$$

It follows that

$$
\lim _{n \rightarrow \infty} \inf \frac{R(t)}{t} \geq \sqrt{\pi M}
$$

Finally, we can give the blowup result.

Proposition 9. If $\delta=1, N \geq 4,1<\gamma \leq 2(N-1) / N$, and $E(0)<0$, then the classical solutions of (1) blow up in finite time.

Proof.

Case $1(\beta=0)$. As before, we have, from Propositions 5 and 7 , that

$$
\begin{aligned}
\ddot{H}(t)= & (4-N) \int_{\Omega} \rho|u|^{2} d x+\left[2 N-\frac{2(N-2)}{\gamma-1}\right] \int_{\Omega} P d x \\
& +2(N-2) E(t) \\
\leq & 0+0+2(N-2) E(t) \\
= & 2(N-2) E(0) .
\end{aligned}
$$

It follows that

$$
H(t) \leq(N-2) E(0) t^{2}+\dot{H}(0) t+H(0) .
$$


Suppose the solutions exist globally; then for sufficient large $t$, we see that $H(t)$ is negative as the leading coefficient of the right hand side of $(60)$ is negative. However, $H(t)$ is nonnegative by definition (35). This is a contradiction. As a result, the solutions blow up in finite time.

Case $2(\beta \neq 0)$. Now (59) becomes

$$
\ddot{H}(t)+\beta \dot{H}(t) \leq 2(N-2) E(0) .
$$

It follows by multipying an integral factor $e^{\beta t}$ on both sides and taking integration that

$$
H(t) \leq A_{1}+A_{2} e^{-\beta t}+\frac{2(N-2) E(0)}{\beta} t
$$

for some constants $A_{1}$ and $A_{2}$. Note that this implies that $H(t)$ is negative for sufficient large $t$ as $\beta>0$ and $(N-$ $2) E(0)<0$. Therefore, the solutions blow up in finite time.

\section{Appendix}

We here complement the proof of Lemma 2 by proving the equality (17); namely,

$$
\int_{\Omega}\left(\frac{1}{2}|u|^{2} \nabla \cdot(\rho u)-u \cdot[\nabla \cdot(\rho u \otimes u)]\right) d x=0 .
$$

Proof. Firstly, by divergence theorem,

$$
\int_{\Omega} \frac{1}{2}|u|^{2} \nabla \cdot(\rho u) d x=-\frac{1}{2} \int_{\Omega}(\rho u) \cdot \nabla|u|^{2} d x .
$$

Secondly, by definitions of the operations,

$$
\begin{aligned}
u \cdot[\nabla \cdot(\rho u \otimes u)] & =\sum_{j=1}^{N} u_{j}\left(\sum_{i=1}^{N} \partial_{i}\left(\rho u_{i} u_{j}\right)\right) \\
& =\sum_{j=1}^{N} u_{j}\left(\sum_{i=1}^{N}\left(u_{j} \partial_{i}\left(\rho u_{i}\right)+\rho u_{i} \partial_{i} u_{j}\right)\right) \\
& =\sum_{j=1}^{N} u_{j}^{2} \sum_{i=1}^{N} \partial_{i}\left(\rho u_{i}\right)+\sum_{j=1}^{N} u_{j} \sum_{i=1}^{N} \rho u_{i} \partial_{i} u_{j} \\
& =|u|^{2} \nabla \cdot(\rho u)+\sum_{i=1}^{N} \rho u_{i} \sum_{j=1}^{N} \frac{1}{2} \partial_{i} u_{j}^{2} \\
& =|u|^{2} \nabla \cdot(\rho u)+\frac{1}{2} \sum_{i=1}^{N} \rho u_{i} \partial_{i}|u|^{2} \\
& =|u|^{2} \nabla \cdot(\rho u)+\frac{1}{2}(\rho u) \cdot \nabla|u|^{2} .
\end{aligned}
$$

Thus,

$$
\begin{aligned}
\int_{\Omega} u \cdot & {[\nabla \cdot(\rho u \otimes u)] d x } \\
= & \int_{\Omega}\left(|u|^{2} \nabla \cdot(\rho u)+\frac{1}{2}(\rho u) \cdot \nabla|u|^{2}\right) d x \\
= & \int_{\Omega} \frac{1}{2}|u|^{2} \nabla \cdot(\rho u) d x . \quad \text { by }(\text { A. } 2) .
\end{aligned}
$$

Thus, equality (A.1) is established.

\section{Conflict of Interests}

The authors declare that there is no conflict of interests regarding the publication of this paper.

\section{Acknowledgment}

This research paper is partially supported by Grant (MIT/SRG02/14-15) from the Department of Mathematics and Information Technology of the Hong Kong Institute of Education.

\section{References}

[1] S. Wong and M. W. Yuen, "Blowup phenomena for the compressible euler and Euler-Poisson equations with initial functional conditions," The Scientific World Journal, vol. 2014, Article ID 580871, 5 pages, 2014.

[2] C. F. Chen, Introduction to Plasma Physics and Controlled Fusion, Plenum Press, New York, NY, USA, 1984.

[3] P.-L. Lions, Mathematical Topics in Fluid Mechanics, vol. 1-2, The Clarendon Press, Oxford, UK, 1998.

[4] B. Perthame, "Nonexistence of global solutions to euler-poisson equations for repulsive forces," Japan Journal of Applied Mathematics, vol. 7, no. 2, pp. 363-367, 1990.

[5] M. W. Yuen, "Stabilities for Euler-Poisson equations in some special dimensions," Journal of Mathematical Analysis and Applications, vol. 344, no. 1, pp. 145-156, 2008.

[6] T. C. Sideris, "Formation of singularities in three-dimensional compressible fluids," Communications in Mathematical Physics, vol. 101, no. 4, pp. 475-485, 1985.

[7] T. Makino, "On a local existence theorem for the evolution equation of gaseous stars," in Patterns and waves, vol. 18, pp. 459-479, North-Holland, Amsterdam, The Netherlands, 1986.

[8] T. Makino, "Blowing up solutions of the Euler-Poission equation for the evolution of the gaseous stars," Transport Theory and Statistical Physics, vol. 21, pp. 615-624, 1992.

[9] T. Makino and B. Perthame, "Sur les Solutions a symmetric spherique de lequation d'Euler- poisson Pour levolution d'etoiles gazeuses, [On radially symmetric solutions of the Euler-Poisson equation for the evolution of gaseous stars]," Japan Journal of Applied Mathematics, vol. 7, pp. 165-170, 1990 (French).

[10] Y. B. Deng, T.-P. Liu, T. Yang, and Z.-A. Yao, "Solutions of euler-poisson equations for gaseous stars," Archive for Rational Mechanics and Analysis, vol. 164, no. 3, pp. 261-285, 2002. 


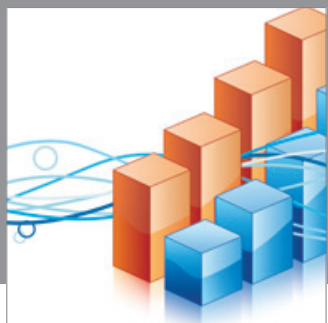

Advances in

Operations Research

mansans

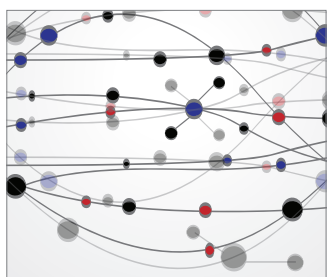

The Scientific World Journal
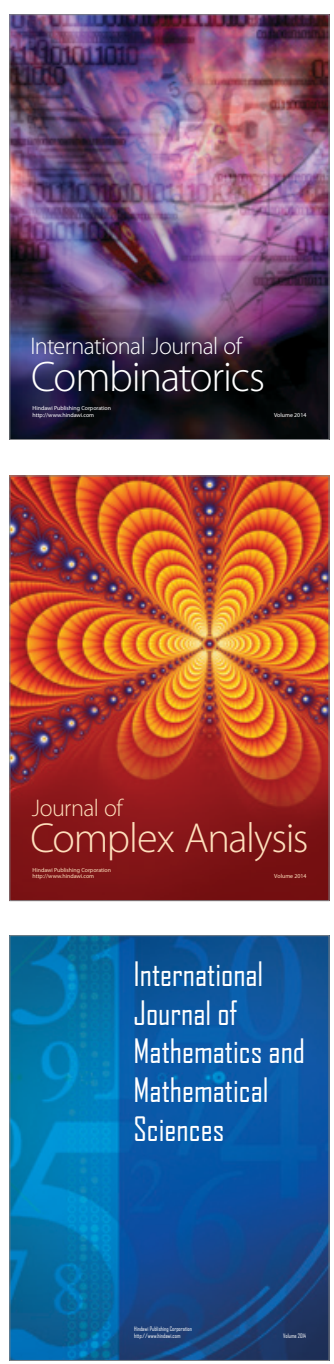
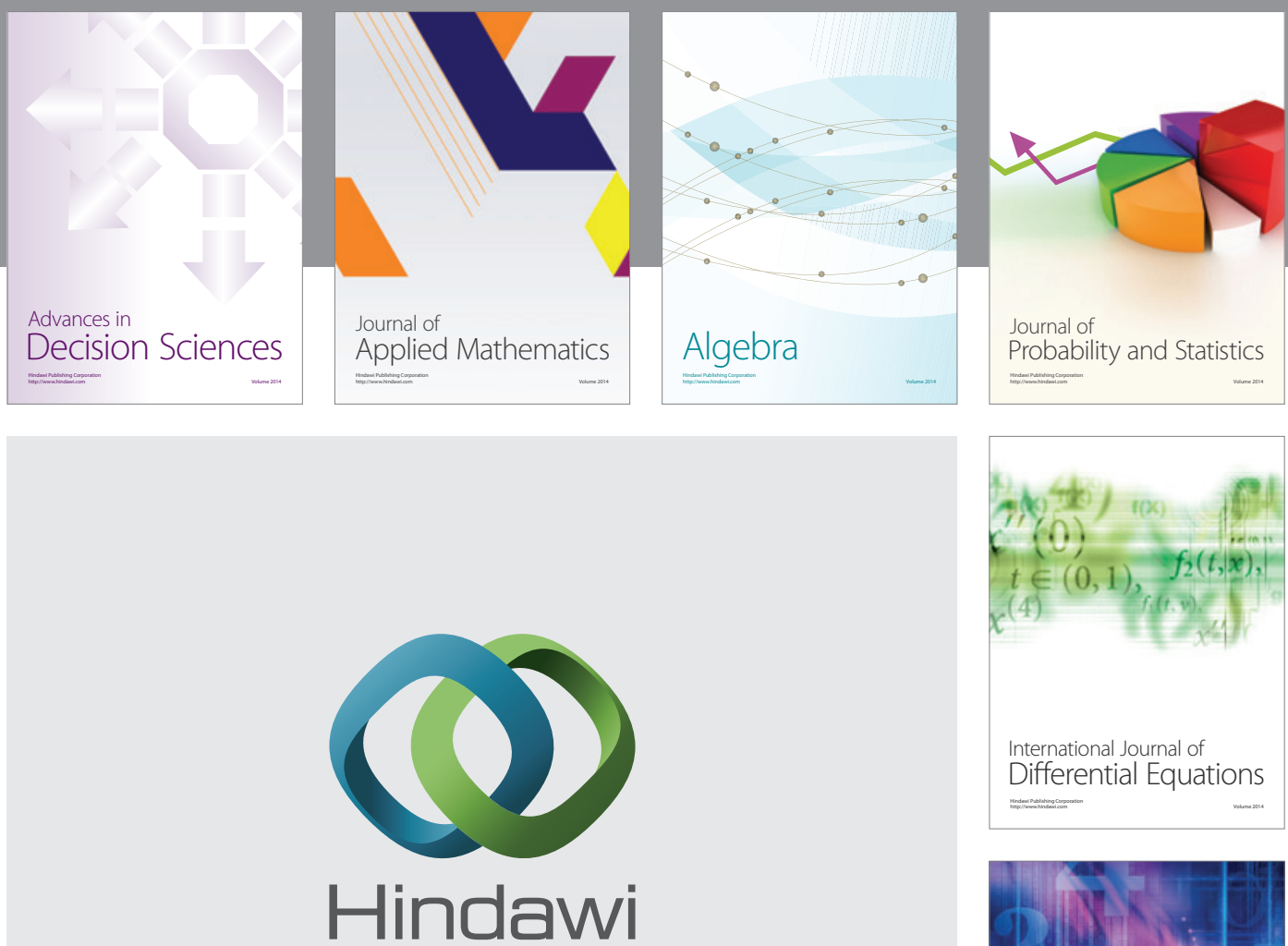

Submit your manuscripts at http://www.hindawi.com
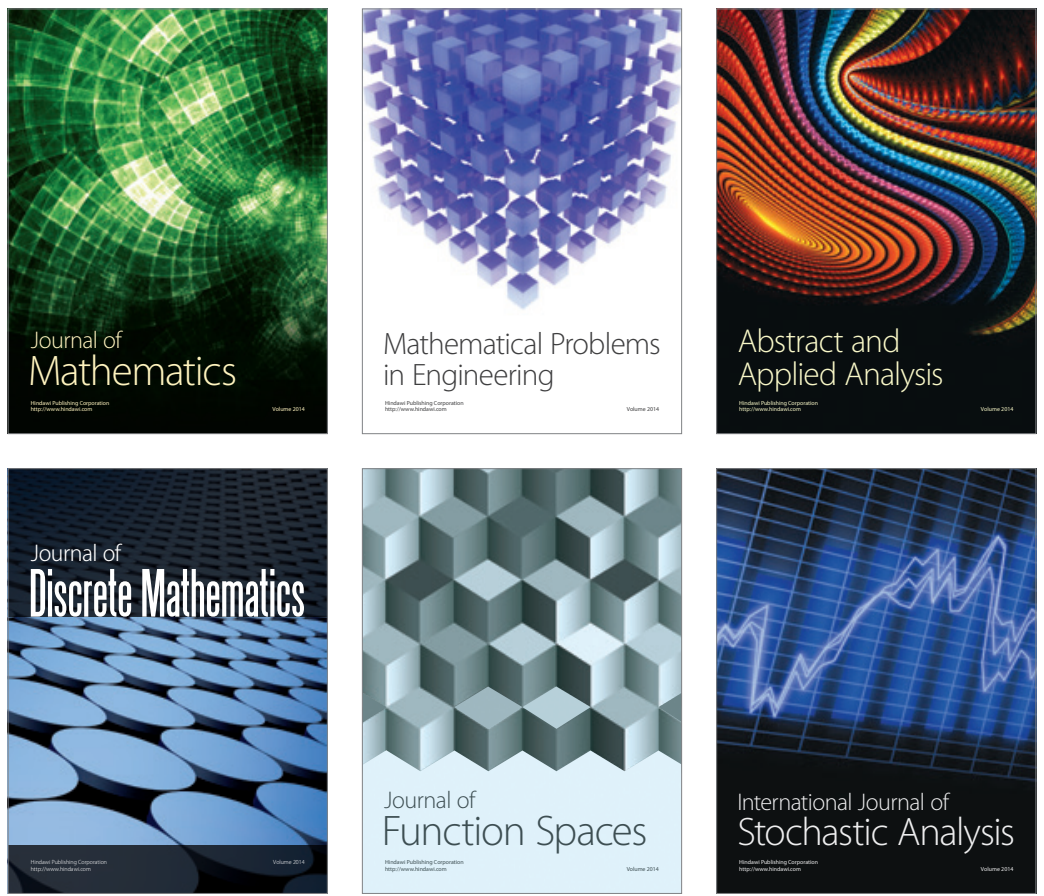

Journal of

Function Spaces

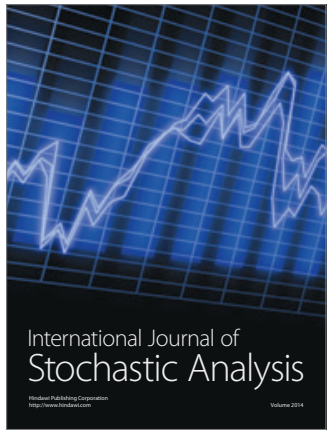

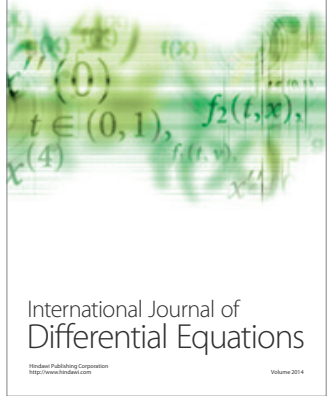
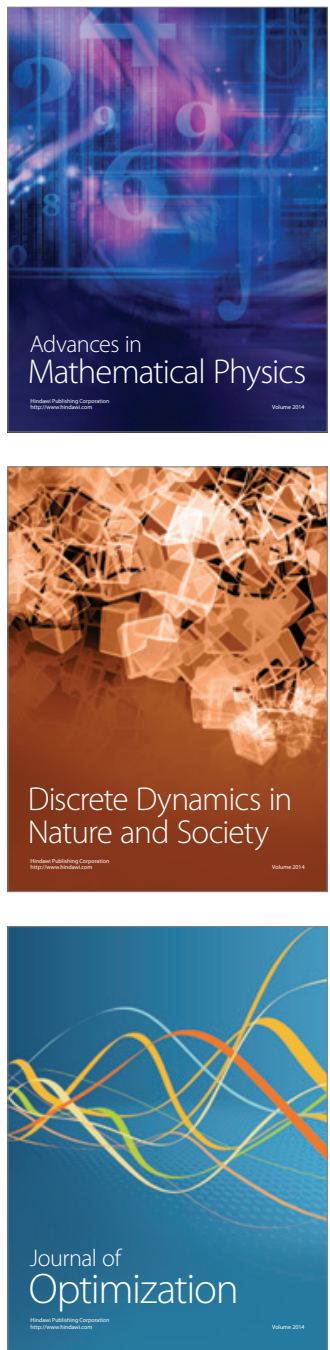